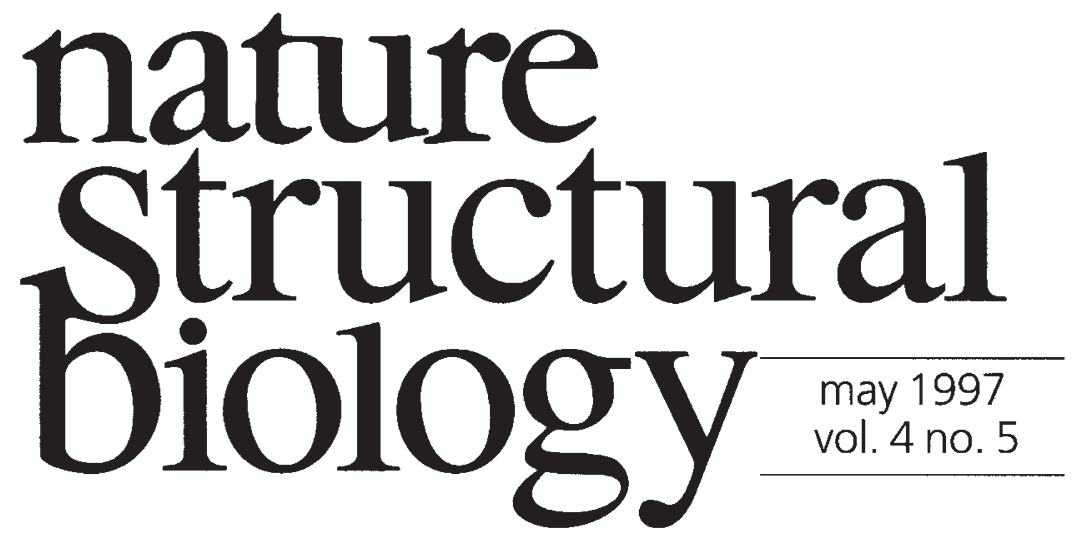

\title{
Structure and the genome
}

“There wasn't a single mention of any structural data!" Janet Thornton's agitated comment regarding the first day of a recent two-day conference on 'genomic medicine' highlights what many perceive as a "rift between the sequence world and the structure world"-Thornton, again (University College, London)and is, perhaps, as much a wake-up call for structural biologists as it is for genome scientists. At least part of the- 'rift' stems from the clash of scientific cultures and the inherent complexity of structure data. As Steve Bryant (National Center for Biotechnology Information (NCBI), National of Health) observes, "even experienced sequence analysts can sort of get ill when they look at a coordinate file".

The issue is not so much with the investigation of individual genes or proteins, or with the process of drug design, where researchers in other fields are well aware of the value of structural information. Rather, with the genome projects in full swing - churning out magabases of DNA sequence-the function of the multitude of ORFs and ESTs being discovered has become a pressing issue: for example, in the case of the yeast genome, less than half of the putative genes identified have an assigned biological role. It is here, as part of the booming business of comparative sequence analysis, that structure should be playing an important role in providing vital clues to function.

Surely, then, there is a premium on maximizing the utility of structural data. The task is not an easy one, though. Three-dimensional structural information "is algorithmically horrendously complicated, requiring real computing and real science" according to Graham Cameron, at the European Bioinformatics Institute (EBI), Hinxton, UK, much more so than the linear arrays of genome sequences, which are merely staggering in their quantity. Furthermore, macromolecular structural information presents special problems in terms of being made readily accessible and comprehensible to the general molecular biologist.

\section{Data bank blues}

The responsibility for the dissemination of such information must fall to the various structure and sequence data banks. But should they also be responsible for providing the software tools that lay biologists need to gain maximum benefit from structural data, especially at a time when many of the data banks are struggling to maintain funding for their core activities ${ }^{1,2}$ ? Cameron believes that "the job of data banks is to provide information, not tools. That should fall to third party software developers."

Indeed, the generally accepted 'official' repository for all atomic resolution 
structural information, the Brookhaven Protein Data Bank (PDB), functions as little more than an archive, as Joel Sussman, its head, acknowledges. His primary goal, he says, must be "validation of the quality of the all structural data" stored at Brookhaven. And although Sussman laments the fact that he does not have the resources to present the data in a more user-friendly fashion, he, like Cameron, feels that this is not part of the mission statement of data banks. Nevertheless, complaints that extracting information from the PDB can be difficult do not fall on deaf ears. Sussman and colleagues are continually working on ways to increase the utility of the PDB.

But what of annotation - or the provision of additional information associated with each item of stored data, be it DNA or protein sequence-in the various data banks? Here is a means by which structural information for a particular sequence can be provided in a comprehensible and easily accessibly manner to the end-user, at relatively little cost to the data providers. Of SwissProt, which is considered one of the best annotated data banks, Thornton complains that it suffers from being sporadically, and not systematically, annotated for structural information. The annotation of the PDB is even more scanty, she adds, with only roughly a sixth of the files having so-called 'site' entries, listing important functional residues such those at the active site.

But this is not to say that structural information is being neglected. One of the strongest, and most explicit attempts to integrate the sequence and structural worlds has been made by the NCBI, and the development of their new browser, Entrez ${ }^{3}$. Features such as their molecular modelling database, and nearest structural neighbouring, goes some way towards providing the kind of support that Sussman is looking for from outside the PDB. The limited compatibility of the data in the various data banks presents another obstacle to more extensive integration. "Data warehousing" says Cameron, "provides an interim solution to the problem": for example, the sequence retrieval system (SRS) developed at the EBI, is a "post facto tool for pulling [data from different sources] into a sump so you can work on them". The long-term solution to the problem is to develop relational, or object oriented data bases, such as the 3DB version of the PDB which, Sussman says, will be available by the end of 1997.

\section{The cultural divide}

"What do molecular biologists fear most? Three letters: PDB". Bryant's joke, told with relish, provides an apposite illustration of the way in which the intricacies and complications of structural information are regarded by some genome scientists. Furthermore, the success of pattern matching at the sequence level has allowed researchers to take a "brainless approach" to comparative analysis, adds Cameron: they don't need to understand that similarity in sequence is generally evidence for similarity in structure (and, therefore, chemistry) to get the right answer, most of the time.

Added to this is the fact that structural biologists are being hard pressed to keep up with the banks of automated sequencers and humming computers grinding away on the various genomes: for example, of the 6195 yeast proteins only $475(7.7 \%)$ have had their three-dimensional structure determined, according to Thornton; the figures for Haemophilus Influenzae (9.3\%) and Mycoplasma genitalium (9.5\%) are not much better. Bryant notes that, for insight into function, it may well be easier for researchers analysing the genome to wait for sequences with a high percentage similarity to appear in one of the data banks than to bother investigating any structural leads.

Is structural information, therefore, largely irrelevant for analysis of the genome? No, the problems simply haven't gotten hard enough yetthus, those data banks that neglect structural data will be doing a disservice to their users.

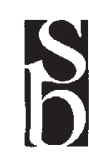

